\title{
Perversion maternelle et troubles du comportement de l'enfant: I'exemple de Dylan, objet du caprice de sa mère*1
}

\author{
Virginie Martin-Lavaud*2
}

\begin{abstract}
À partir d'une situation clinique, nous analysons en quoi les troubles dits du comportement de l'enfant viennent répondre à une position psychique perverse maternelle. Nous mettons l'accent sur la notion de fantasme maternel odipien mis en acte et questionnons la position d'objet fétiche de l'enfant. Nous montrons notamment que le statut des actes du sujet pris dans le non-sens et la tromperie, objet du caprice maternel, désigne un désir inscrit hors la loi phallique et que cette inscription est en soi perverse. Elle explique que l'enfant n'a alors pas d'autre alternative que de devenir lui-même clivé et hors sens.
\end{abstract}

Mots clés: Perversion maternelle, troubles du comportement de l'enfant, mère-version, rapport aphallique, psychopathie

*1 Texte présenté au colloque de 1'Universidade Católica de Pernambuco - Unicap sur Les usages sociaux de la perversion, Recife 26-28 août 2013, Brésil.

*2 Université Catholique de l'Ouest (Angers, France). 


\section{Introduction}

La question des troubles du comportement de l'enfant est toujours plus ou moins d'actualité dans les écoles primaires en France. Elément symptomatique de l'évolution de la structure familiale, cette question introduit à la fois le rôle symbolique des parents dans l'éducation de leurs enfants et leur position imaginaire dans le psychisme. Notre pratique clinique de psychologue, psychanalyste, nous amène à rencontrer des enfants qui se mettent hors-circuit. Or nous faisons le constat qu'ils soient filles ou garçons, que ces enfants dont la violence verbale, physique et les conduites transgressives déstabilisent les enseignants, n'existent souvent qu'en référence à leur mère. Cette remarque ne veut pas dire que le père est absent dans la réalité. Ce que nous impliquons est que le père est tenu à distance dans le discours maternel et que l'enfant n'a pas d'autre choix que d'en prendre acte. Ce pouvoir de la mère sur l'enfant n'est pas sans avoir d'effets pervers. Lacan l'avait également noté et le formulait ainsi: «s'il y a moins de perversions chez les femmes que chez les hommes, c'est qu'elles satisfont, en général, leurs rapports pervers dans leurs rapports avec leurs enfants» (Lacan, 1959-1960/1986, p. 530). Ci-après nous allons, en nous appuyant sur le suivi d'un enfant que nous avons nommé Dylan, articuler la question du destin du désir maternel et les conditions de son retournement en perversion, retournement dont nous pourrons discuter de la position de structure ou du simple versant ${ }^{1}$ (Lebrun, 2005a, p. 228).

${ }^{1}$ Lebrun l'écrit ainsi: «Il ne nous est donc pas difficile de penser que la prévalence octroyée aujourd'hui au régime de la relation à la mère — parce que le patriarcat n'exerce plus sa contrainte de force motrice pour la subjectivation - incite au modèle pervers dans la mesure où nous faisons de celui-ci précisément ainsi que Lacan nous y invite, l'impasse du registre imaginaire. Néanmoins reste la question de savoir s'il s'agit pour autant de véritable perversion au sens où l'on entendrait une structure perverse. Il faut ici d'emblée introduire un discernement de taille, à savoir qu'il nous faut distinguer entre la position de force de l'identification qui donne son assise au sujet pervers et cette position de prédominance perverse — au sens de récusation de l'asymétrie et de l'hétéronomie — que l'on pourrait dire artefactuelle qui permet au sujet de faire objection au travail de subjectivation, parce que le contexte social lui permet de reporter sans cesse la confrontation à la différence des places» (Lebrun, 2005a, p. 228). 


\section{Présentation de l'histoire de Dylan}

Nous avons fait la connaissance de cet enfant après un appel au secours de son école: il venait de gifler la maîtresse remplaçante. Lorsque nous l'avons reçu, Dylan était âgé de sept ans. Intelligent, il disait souhaiter changer de comportement afin de ne plus être victime de sa propre colère. Il avait repéré que dès qu'il se sentait critiqué, il tapait ses camarades et que lorsqu'il refusait les sanctions tout empirait car, se faisant emporter par ses émotions, il insultait les adultes responsables de lui, à l'école, comme à la maison. Dylan était l'unique enfant de sa mère, laquelle avait quitté son père après différents épisodes de violence conjugale dont elle ne nous donnera pas les motifs. Lorsque nous ferons sa connaissance, elle était remariée à un homme dont elle n'avait pas d'enfant et qui lui-même n'était pas père. Très rapidement nous apprendrons, par le directeur de l'école, que la relation que cette femme entretenait avec son fils était ambiguë car faite de cajoleries et de baisers ou de réprimandes et de violence verbale. Les enseignants en étaient témoins impuissants car ils assistaient à ces scènes lorsque cette mère emmenait son enfant à l'école. L'excès de l'ambivalence affective mère/fils se révéla notamment un matin lorsque cette femme furieuse contre lui menaça de le tuer puis de se tuer en retour. Le ton et la conviction avec lesquels elle formula ses menaces, furent suffisamment inquiétants pour que le directeur en fasse part à sa hiérarchie et rédige un compterendu à destination du juge des enfants.

Ce fonctionnement relationnel clivé, organisé par le «tout ou rien» de l'amour, Dylan n'en était pas dupe. Certes il le subissait mais il en tirait également une certaine jouissance. Il verbalisera cet état de fait en indiquant que sa mère lui reprochait de perturber son couple et que si elle devait divorcer, ce serait à cause de lui. Sa place et son désir à l'égard de sa mère étaient donc également ambiguës. Il nous le soulignera en nous indiquant notamment posséder un savoir sur l'intimité sexuelle de sa mère qu'il n'aurait pas dû détenir. Il savait que sa mère ne pouvait plus concevoir parce qu'elle n'avait plus d'ovules. Ce savoir sur la sexualité de sa mère inscrivait de fait les relations sexuelles de cette dernière avec son mari dans un registre qui n'était pas de l'ordre de la procréation et éclairait d'une lueur transgressive incestuelle la place de Dylan vis-à-vis d'elle. D'une certaine manière ce savoir ajoutait une touche exhibitionniste aux baisers et étreintes que mère et fils partageaient avant de se séparer lorsqu'elle l'emmenait à l'école.

$\mathrm{Au}$ retour d'une période de vacances scolaires, les enseignants ne reconnurent pas la mère de l'enfant: elle avait énormément maigri et était d'une humeur noire. Un mois après ce descriptif, son mari se suicida par pendaison; acte par lequel en «faisant veuve» sa femme, cet homme inscrivit son destin en place de conjoint à vie dans la mesure où ce geste lui conférera dans l'après-coup la place du mari pour 
l'éternité2 (Lacan, 1957-1958/1998, p. 245), place symbolique qui lui faisait défaut dans la réalité. Sur le moment, Dylan ne dira rien du suicide mais quatre à cinq mois plus tard, il s'en accusera. Ce retour de la culpabilité généra une montée des conduites violentes, lesquelles l'isolèrent encore plus des autres enfants et provoquèrent l'exclusion définitive de son école pour une autre. Dans cette nouvelle structure, la narration de tous ces événements l'avait précédé. Il ne réussira jamais à se faire d'amis. Il fera même éclater le contexte institutionnel en refusant d'entrer en classe et là encore, en insultant tous les adultes responsables, en les menaçant avec des ciseaux et en détruisant le matériel. L'administration de l'éducation nationale ne pouvant juridiquement l'exclure sera contrainte de lui attribuer un précepteur qui lui fera cours jusqu'aux vacances d'été, avant qu'il ne soit orienté en internat, à la demande de sa mère, dans une structure spécialisée dans l'accueil d'enfants dits caractériels.

On peut d'ores et déjà remarquer que Dylan par ses provocations obtint un adulte enseignant pour lui seul; un adulte avec lequel tout se passait bien. Il n'était plus dans l'excès alors qu'il était toujours dans le cadre scolaire. On peut se demander si ses débordements n'avaient finalement pas pour fonction d'appeler un père symbolique, un représentant du savoir sur la loi. Précisons pour conclure l'exposé du contexte, que le suicide du beau-père révéla dans l'après-coup l'existence d'un personnage jusqu'alors resté dans l'ombre: le grand-père maternel. Ce dernier entra en scène sous la forme du vociférateur car il injuria et menaça le directeur de la nouvelle école, comme sa fille et son petit-fils l'avaient maintes fois fait avant lui. Cela se produisit lorsque ce directeur convoqua la mère pour lui indiquer que Dylan ne pouvait plus être accueilli dans son établissement en raison de la violence de ses actes et de son inaccessibilité à la demande de l'autre en position d'autorité.

Les principaux protagonistes de l'histoire étant maintenant tous mis en lumière: le grand-père maternel, la mère et l'enfant, que pouvons nous en déduire.

\section{L'agir du fantasme et ses implications}

Ici l'organisation familiale fait nettement apparaître une logique centrée autour du désir de la mère. Le père de l'enfant et le beau-père sont très absents et

${ }^{2}$ «Plus le sujet s'affirme à l'aide du signifiant comme voulant sortir de la chaîne signifiante, et plus il y entre et s'y intègre, plus il devient lui-même un signe de cette chaîne. S'il s'abolit, il est plus signe que jamais. La raison en est simple — c'est précisément à partir du moment où le sujet est mort qu'il devient pour les autres un signe éternel, et les suicidés plus que d'autres» (Lacan, 1957-1958/1998, p. 245). 
leur parole peu soutenue par la mère. Cette mère, dans la réalité, n'occupait d'ailleurs pas réellement une place maternelle puisqu'elle s'exhibait à l'école soit comme séductrice, soit comme tyran de son enfant. L'apparition du grand-père, après la mort du mari, sera le point conclusif d'une mise en scène dont la logique apparaît perverse tant elle met en avant des «agir» transgressifs du côté de l'enfant et un «agir» du fantasme œdipien du côté maternel. Psychiquement, on peut remarquer que cet enfant occupe une place étrange dans le désir de sa mère; une place qui n'est pas celle de la logique ternaire que propose Lacan dans son quatrième séminaire: mère, enfant, phallus. En fait la dynamique familiale de Dylan est essentiellement marquée par une logique d'emboîtement, comme celle des poupées russes; une logique structurée par un rapport de domination: le grand-père dominant la mère qui domine l'enfant, laquelle utilise son enfant pour satisfaire sa jouissance sur un mode infantile. Certes la question phallique n'est pas absente mais elle est voilée par la mise en acte d'une parole qui annule l'ordre des générations au profit de la transgression de l'interdit œdipien. Cet agir du fantasme, parce qu'il appelle la réalisation du rapport sexuel ${ }^{3}$ (Lacan, 1979, p. 8) met cependant en question le statut même de la logique perverse dans le désir de la mère. En effet doit-on considérer qu'il s'agit d'une perversion, c'est-à-dire d'une logique organisée par le déni de la castration de la mère, pèreversion, ou comme le proposent Jean-Pierre Lebrun (2005b) ou Julia Kristeva ${ }^{4}$ (2011), d'une mère-version, c'est-à-dire d'une logique organisée par un rapport aphallique à la mère motivée soit par une dynamique phobique à l'égard de l'objet phallique, soit par une construction de l'autre et l'objet qui serait «en-deçà du stade du miroir» (Kristeva, 2011, p. 1565) et donc proche de la Chose.

Nous savons en effet que dans la perversion, c'est le rapport à la castration de la mère qui est central, le rapport à l'objet phallique, alors que dans la mère-version, ce qui est mis en avant serait une forme de perversion infantile, c'est-à-dire non organisée par le signifiant phallique, une version presque phobique à l'égard du phallus, qui s'arrimerait sur le fantasme, non pas refoulé mais agi, comme peut le faire tout enfant qui explore les voies de la pulsion. Si nous poursuivons l'idée d'une

${ }^{3}$ «Le rapport sexuel, il n'y en a pas, mais cela ne va pas de soi. Il n'y en a pas, sauf incestueux. C'est très exactement ça qu'a avancé Freud — il n'y en a pas, sauf incestueux, ou meurtrier. Le mythe d'Edipe désigne ceci, que la seule personne avec laquelle on ait envie de coucher, c'est sa mère, et que pour le père, on le tue» (Lacan, 1979, p. 8).

${ }^{4}$ «Cet appétit d'excitation, continûment à la recherche d'un objet de satisfaction lui-même continûment insatisfaisant, serait donc l'inévitable destin de la pulsion chez les humains, la véritable économie de ce que Freud a repéré comme une perversion originelle. Et de se demander si la notion même de perversion n'est pas un concept «contre-phobique», et si la perversion ne recouvre pas plutôt une... «mère-version»! J. Kristeva reprend cette formulation chez Isle Barande, propos tenus dans le cadre de son (Kristeva, 2012). 


\section{ARTIGOS}

mère-version, on peut dire que le mode de jouissance de la mère de Dylan est infantile et qu'il inscrit son enfant à une place étrange, une place qu'il nous faut définir.

Cette place est-elle celle d'un objet fétiche, d'un objet phallique, d'un objet caprice? Nous avons vu que la monstration des manifestations affectives inadéquates décrites par les enseignants entre cette mère et son fils, leurs étreintes et leurs conflits attestent du fait que pour cette mère l'enfant est une sorte de partenaire amoureux. A partir de cette observation, nous avons privilégié l'idée qu'il était un objet caprice de la mère plutôt qu'un objet fétiche; l'objet fétiche étant par définition au service de l'illusion comme l'indique son étymologie portugaise fétiçio: il vient faire «illusion à». Ici Dylan n'illusionne pas la mère sur son manque d'objet puisqu'elle ne manque de rien: elle est comblée par l'amour adressé à son propre père. De ce fait Dylan est à la place d'un objet en trop, d'un objet de l'excès.

Ce prolifique, cet «en trop» signifie-t-il que cet enfant serait au service d'une jouissance qui ne serait pas toute phallique?

Cette proposition s'appuie sur le fait que pour exister dans le désir de sa mère Dylan s'offre à elle par identification. L'événement de la gifle en témoigne notamment car lorsque Dylan gifla la maîtresse remplaçante, son geste fut précédé d'un acte violent imprévu dont le jeu du signifiant introduit une jouissance Autre, celle que Lacan situe à la jonction des registres du réel et de l'imaginaire. Ainsi, alors qu'il se débattait pour échapper à son enseignante qui venait de le réprimander, il se fit mal au pied en heurtant le pied d'un bureau qui cassa sous le choc. Or, dans la réalité, à la même période, sa mère avait également le pied cassé car elle s'était (aussi) blessée sur son lieu de travail. Ce curieux mouvement d'identification dans lequel la mère comme le fils se trouvent blessés au pied introduit une sidération imaginaire dans laquelle le signifiant est comme englué dans la jouissance. Alors que tous deux se trouvèrent représentés par le fait d'être dérangeants et indésirables pour les autres — ils étaient identifiés au signifiant «casse-pied» — ils se trouvèrent marqués sur leur corps au même endroit. Bien évidemment ce pied de la lettre n'est pas sans faire jouis-sens.

\section{Peut-on conclure que les troubles du comportement de cet enfant venaient en réponse à une logique perverse dans laquelle il n'avait qu'une place par défaut?}

En adjoignant la jouissance à la «satisfaction d'une pulsion» et non d'un besoin (Lacan, 1959-1960/1986, p. 247-248), Freud (1905/1987) précise que la recherche de jouissance est au service de la nostalgie d'un temps avant la perte de l'objet, un temps avant la confrontation à la castration. C'est pourquoi l'objet pulsionnel n'est jamais qu'un objet retrouvé (Wiederfindung) au service de la répétition (Wiederholungswang), un objet auquel l'existence de l'enfant peut être conjointe lorsque l'enfant est assimilé à un objet répondant aux caprices du désir de la mère. Lorsque l'enfant répond à cette place, c'est qu'il est assimilé à l'objet à 
exclure, à l'objet abject que l'on prend et que l'on jette, à la guise de son désir. Julia Kristeva (1980) dans son ouvrage Pouvoirs de l'horreur conceptualise justement la question de l'abjection en indiquant qu'elle introduit une jouissance Autre, une jouissance qui échappe à la logique phallique dans la mesure où l'abjection rend compte de l'insignifiable, de ce qui précède le narcissisme dont elle est une pré-condition. Elle précise: «l'abject nous confronte (...) à nos tentatives les plus anciennes de nous démarquer de l'entité maternelle avant même que d'ex-sister en dehors d'elle grâce à l'autonomie du langage» (Kristeva, 1980, p. 20).

\section{Un fils en trop, un fils hors-norme?}

La perversion parce qu'elle a la «propriété de réaliser un mode d'accès à cet au-delà de l'image de l'autre qui caractérise la dimension humaine» (Lacan, 1956, p. 85) introduit la dimension du hors-norme et de l'anormalité, laquelle s'accorde point par point avec le signifiant «monstre». Ce dernier désigne esthétiquement et logiquement les entités floues, mal définies (Martin-Lavaud, 2009a, 2013). Or dans le regard des autres Dylan était à cette place; il en revêtait plusieurs aspects car il était perçu à la fois comme imprévisible et effrayant mais également comme a-sexuée. Il nous en parla un jour en nous expliquant qu'il s'était mis en colère le jour où une enfant de son école lui dit qu'il riait comme une fille. Le pointage de l'ambigüité de sa position sexuée, lui qui pensait être vu comme détenteur du phallus parce qu'il posait des actes violents, engagea sa réflexion sur son être. Certes «être un monstre» n'était pas en soi une position que Dylan revendiquait mais c'était une image qu'il renvoyait et qui résumait finalement assez bien quelle était la scène sur laquelle cet enfant était inconsciemment joué. D'une certaine manière, en tant qu'objet déphallicisé dans le désir de sa mère, Dylan était inscrit dans une position invivable, une position de grande solitude, telle celle du monstre produit par le Dr Frankenstein dans le roman de Mary Shelley qui se plaint justement de son isolement. La figure du monstre, en tant qu'entité permettant d'esthétiser et de mettre en question le statut du phallus, oriente le sens de l'acte du côté de l'éthique, ${ }^{5}$ ce que questionnait également Dylan en séance lorsqu'il réalisait l'anormalité de ses colères, de ses humeurs et de ses réactions. Il l'exprima un jour dans ses dessins en se représentant « hors de lui », extérieur à lui-même. Cf. Figures 1 et 2.

${ }^{5}$ «Pour le sujet pervers, le monstre n'a généralement d'intérêt et d'existence que lorsqu'il y est identifié, lorsqu'il se sait désigné comme tel par les autres. C'est la raison pour laquelle le monstre esthétise les clivages du moi, la jouissance, la pulsion, l'existence de zones d'ombres de la personnalité, et rend compte de la «contre-nature» ou «contre logique» des actes» (Martin-Lavaud, 2009b, p. 202). 


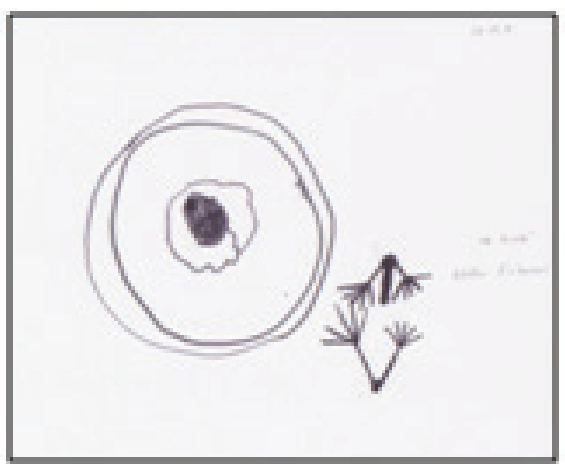

Figure 1

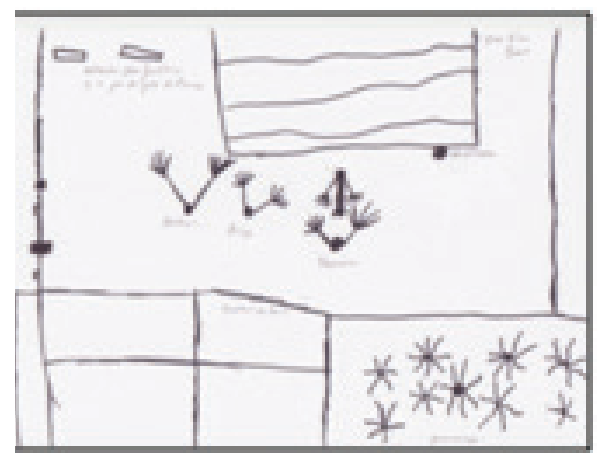

Figure 2

\section{Être hors de soi: une position d'ex-sistence}

Cette mise en représentation de lui-même, témoin de la subjectivation de sa parole et de sa vision du monde, fait écho au fait d' "être hors de soi», qui en français signifie "être en colère»: laisser éclater sa colère était d'ailleurs le symptôme le plus criant de son mal-être. Sa mère l'avait pour cela conduit deux ou trois fois chez un psychothérapeute hypnotiseur, non contente du peu de changement de la cure avec moi-même. Elle dit après deux séances: «il a fait des miracles, il lui a pris sa colère», son fils ajoutant: "j'ai une colère en moins, je me sens bien.» Le répit fut cependant de courte durée. Dylan n'était pas sans savoir qu'en disant qu'il en avait une en moins, une seulement, il en restait encore un certain nombre à soustraire.

La constatation de sa solitude amena cet enfant à interroger la question de l'amour. Il se demanda pourquoi les autres avaient des amis et comment il devait s'y prendre pour s'en faire. Chez lui, il passait son temps à jouer avec ses chiens, à être leur maître et les battait lorsqu'ils n'obéissaient pas. Cette recherche de maîtrise était d'ailleurs également à l'œuvre dans sa propension à agresser les autres. Il voulait avoir le dernier mot. Ainsi tentait-il de se rassurer en s'attribuant une puissance phallique, dont il reconnaissait également l'illusion. Loin d'être psychotique, Dylan était marqué par la castration, mais il ne parvenait pas à l'articuler pour qu'elle prenne un sens qui lui permette d'accueillir l'autre. Il disait volontiers pour se rassurer: «quand je n'aime pas les gens, ça va mal.»Sa mère confirmant que «le mot «non» il ne faut pas l'employer, sinon ça part à tout va». 


\section{La psychopathie comme solution pour ex-sister}

Cet enfant utilisait donc la violence des actes et des mots non pas pour se convaincre qu'il n'était pas castré, ce qu'il devinait sans en définir les conditions, mais pour exister contre le désir pervers maternel. Il s'en défendait comme un beau diable, position qui n'est pas sans faire écho à celle du psychopathe. "Le psychopathe est étymologiquement celui qui souffre dans sa psyché» Chartier, 2007, p. 62) ${ }^{6}$ ce que nous n'associons pas à une position perverse.

A l'école, les conduites de Dylan étaient psychopathes. Elles étaient données à voir comme signe d'une inscription imaginaire phallique laquelle, nous l'avons expliqué, révélait d'autant plus son défaut d'inscription phallique symbolique dans le désir de sa mère. En contrepartie, cet enfant était très soucieux de ce que les autres lui renvoyaient narcissiquement, au point de s'en rendre «malade». Il était comme englué dans son narcissisme. ${ }^{7}$ Un jour que son enseignante avait affiché au tableau, pour elle-même y penser, le rendez-vous que j'avais donné à Dylan, ce dernier m'en parla en me disant qu'il ne voulait pas que les autres sachent où il allait lorsqu'il s'absentait. Ainsi me signifia-t-il qu'il ne souhaitait pas que son rendez-vous soit divulgué. Cf. Figure 3

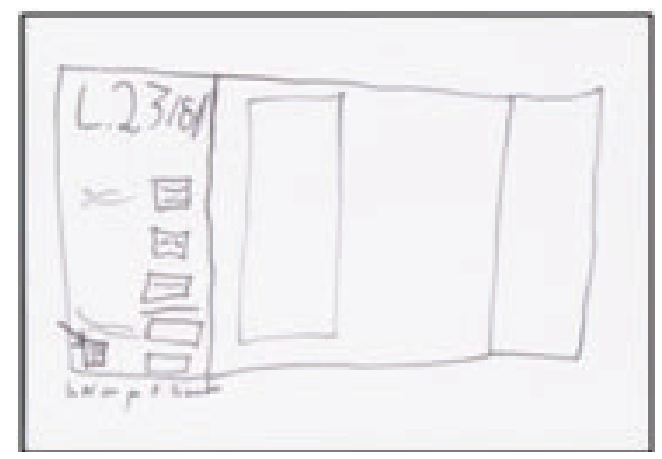

Figure 3

${ }^{6}$ Il précise «En 1923 Kurt Schneider reprendra à Kraepelin le terme de psychopathie pour désigner des états qui ne relèvent en rien de processus organiques pathologiques mais qui dépendent de personnalités «anormales» telles que leur caractère anormal les fait pâtir ou fait pâtir la société. Le psychopathe est étymologiquement celui qui souffre de sa psyché» (Chartier, 2007, p. 62).

${ }^{7}$ «Le narcissique (...) apparaît actif pour autant que, jusqu'à un certain point, il méconnaît l'autre» (Lacan, 1956-1957/1994, p. 83). 


\section{ARTIGOS}

Un autre événement caractéristique de sa sensibilité narcissique fut la remarque de la petite fille sur son rire. Il entendit dans cette remarque qu'il était désigné en position féminine. ${ }^{8}$ On peut d'ailleurs se demander si, pour échapper à la logique perverse du désir de sa mère, Dylan n'en était pas réduit à s'offrir en position féminine, se faisant «enfant à battre» pour ne pas être l'enfant imaginaire donné au grand-père et ce d'autant plus que la fustigation ${ }^{9}$ était une thématique très présente dans son histoire. Elle l'était sous deux aspects: l'un concernait les dires de sa mère au sujet de son père; elle racontait volontiers que son premier mari l'avait battue et que son fils en avait été témoin oculaire à l'âge de trois ans - elle supposait d'ailleurs que cette scène était à l'origine des désordres comportementaux de son fils - le second était que son fils battait également volontiers ses chiens et les autres, souvent sans raison, pour le plaisir de bousculer ou d'être désigné comme terreur. Lacan commentant le fantasme «un enfant est battu» qui intervient avant l'Edipe, souligne que «c'est au niveau du père que se situe sa signification. Le père refuse, dénie son amour à l'enfant battu, petit frère ou petite sœur. C'est pour autant qu'il y a dénonciation de la relation d'amour et humiliation, que ce sujet est visé dans son existence de sujet. Il est l'objet d'un sévice, et ce sévice consiste à le dénier comme sujet, à réduire à rien son existence comme désirant, à le réduire à un état qui tend à l'abolir en tant que sujet» (Lacan, 1957-1958/1998, p. 238). Le sujet dès lors est castré par la loi du père. Or en énonçant devant son fils, le fait d'avoir été battu par le père de ce dernier, la mère signifie donc que son mari ne l'aimait pas justifiant son droit de se re-tourner vers son propre père. On peut également en conclure que pour Dylan l'imaginaire attaché au père est un imaginaire sadique dans la mesure où il est désigné comme celui qui bat sa femme. Peut-on penser que pour Dylan son père de la réalité ne pouvait donc venir en place de père symbolique parce qu'il en était empêché par sa mère qui le rabaissait dans cette position d'agresseur? C'est en effet en tant que père symbolique que le père bat l'enfant, pour le castrer de son désir œdipien et ainsi obtenir sa soumission à la loi.

${ }^{8}$ Rappelons que «pour Freud, ce qui permet de caractériser la structure même de la perversion est donc bien la position du sujet dans le fantasme, et non pas simplement le type de l'objet choisi. On peut déjà repérer que le sujet se féminise dans son identification à la mère. Quant à l'objet, il est un double narcissique du sujet. Ne voit-on pas, dès cette époque, déjà posée en germe la division subjective caractéristique du sujet dans la perversion? Cette hypothèse donne un tout autre sens à l'auto-érotisme que Freud reconnaît dans les perversions, et nous permettra ultérieurement de mieux comprendre quel rôle est attribué à l'objet par le sujet pervers» (Valas, 1986, p. 38).

${ }^{9}$ «La fustigation n'attente pas à l'intégrité réelle et physique du sujet. C'est bien son caractère symbolique qui est érotisé comme tel, et ce dès l'origine» (Lacan, 1957-1958/1998, p. 242). 
Pour se défendre contre cette logique mortifère, Dylan rabaissait lui-même souvent les objets qu'il aurait pu aimer: «la nouvelle maîtresse est nulle, les activités sont nulles et les maîtresses ne servent à rien.» Cette logique destructrice qui met en avant la haine plutôt que l'amour, était également présente dans le transfert: dans un moment de colère, il nous adressa: «vous ne m'avez servi à rien», parole qui était celle de sa mère et qu'il prit à son compte nous laissant entendre que $s a$ parole n'était pas suffisante pour mobiliser le changement, comme si lui-même n'avait pas «droit au chapitre» et était inexistant.

\section{Conclusion}

Pour conclure précisons que le caractère psychopathe du comportement de Dylan ne signifiait pas qu'il soit pervers. Dans un certain sens, au contraire, il s'affirmait comme castré et névrosé, victime de l'Autre et de sa loi puisqu'en faisant état de son mal-être et en nous signifiant notre inutilité, voire notre impuissance, il nous indique qu'il n'est pas vierge de toute considération sur la castration. D'un autre côté, il nous indique également qu'il échouait lui-même à être puissant et donc à se faire objet de la jouissance de l'Autre. On peut ici conclure qu'il reconnaissait qu'il échouait à être pervers. En fait, cet enfant était aux prises avec des questions existentielles et commençait à les articuler pour se dégager de la perversion maternelle.

L'actualité de telles situations cliniques est loin d'être marginale. C'est pourquoi nous estimons essentiel de rappeler que les troubles du comportement des enfants, fréquents en milieu scolaire dans la mesure où l'école appelle le savoir et fait référence à la position symbolique du père, sont très souvent corrélés à une logique perverse $d u$ désir maternel. Certes la question du statut pervers du désir se pose cependant: est-il de structure ou juste un versant du désir? Ce que nous pouvons remarquer s'agissant de la mère de Dylan, c'est qu'elle retirait sa jouissance de faire exister le rapport sexuel, c'est-à-dire, pour reprendre la proposition de Lacan, de s'offrir à son père, comme objet, en-dehors de la loi de l'interdit de l'inceste et que dans cette mesure elle s'offrait en place d'objet de la jouissance de l'Autre tel que le fait le pervers.

\section{Références}

Chartier, J-P. (2007). Peut-on guérir le psychopathe? Topique, Paris, II(99).

Freud, S. (1987). Trois essais sur une théorie de la sexualité. Paris: Gallimard, 1987. (Trabalho original publicado em 1905). 


\section{ARTIGOS}

Kristeva, J. (1980). Pouvoirs de l'horreur, essais sur l'abjection. Paris: Seuil.

Kristeva, J. (2011). La reliance, ou de l'érotisme maternel. Revue française de psychanalyse. Paris, PUF, $V(75), 1559-1570$.

Kristeva, J. Séminaire doctoral, Université Paris 7, Paris-Diderot, Dépression, Perversion, Sublimation, 2012. Recuperado de: <http://www.kristeva.fr/depression-perversion-sublimation.html>.

Lacan, J. (1973). Le séminaire. Libre IX. Les quatre concepts fondamentaux de la psychanalyse. Paris: Seuil. (Trabalho original publicado em 1964).

Lacan, J. (1979). L'escroquerie psychanalytique. Ornicar? Revue du champ freudien, Paris, (17-1), 7-23, leçons du 15 mars 1977.

Lacan, J. (1986). Le séminaire. Libre VII. L'éthique de la psychanalyse. Paris: Seuil. (Trabalho original publicado em 1959-60).

Lacan, J. (1994). Le séminaire. Libre IV. La relation d'objet. Paris: Seuil.. (Trabalho original publicado em 1956-57).

Lacan, J. (1998). Le séminaire. Libre V. Les formations de l'inconscient. Paris: Seuil. (Trabalho original publicado em 1957-58).

Lebrun, J-P. (2005a). Une perversion ordinaire. La clinique lacanienne, Toulouse: Erès, II(9), 215-235.

Lebrun, J-P. (2005b). Éloge de la perversion. Le Coq-héron, Toulouse: Erès, IV(183), 42-58.

Lebrun, J-P. (2012). Lacan et ses états limites. Connexions, Toulouse: Erès, I(97), 77-92.

Martin-Lavaud, V. (2009a). Le monstre dans la vie psychique de l'enfant. Toulouse: Erès.

Martin-Lavaud, V. (2009b). Le monstre comme a-bord du réel dans la clinique de l'enfant. Réel et réalité. Paris: Desclée de Brouwer.

Martin-Lavaud, V. (2013). Le monstre, une altération esthétique pour penser l'humain. Topique, Paris, (122), 83-91.

Valas, P. (1986). Freud et la perversion. (Mémoire de DEA). Département de psychanalyse, Université Paris VIII, p. 38. Recuperado de: <http://www.valas.fr/IMG/pdf/Freud_et_la_perversion_I_II_III.pdf>.

\section{Resumos}

(Perversão materna e problemas do comportamento da criança: o exemplo de Dylan, objeto do capricho de sua mãe)

A partir de uma situação clínica, nós analisamos em que os problemas ditos do comportamento da criança vêm responder a uma posição psíquica perversa materna. Nós destacamos a noção de fantasma materno edipiano posto em ato e questionamos a posição da criança de objeto fetiche. Mostramos particularmente que o estatuto dos atos do sujeito tomados no fora do sentido e na enganação, objeto do capricho 
materno, designa um desejo inscrito fora da lei fálica e que essa inscrição é em si perversa. Ela explica que a criança não tem, então, outra alternativa, que não a de se tornar ele mesmo clivado e fora do sentido.

Palavras-chave: Perversão materna, problemas do comportamento da criança, mãeversão, relação afálica, psicopatia

(Maternal perversion and behavioural disorders in children: the example of Dylan, an object of his mother's whims)

Based on a clinical case, we studied how so-called behavioral disorders in a child correspond to perverse states of the mother's mind. We show Oedipal fantasies of the mother and see the child's possible position as a fetish. We particularly show that the status of the subject's actions caught up in nonsense and deceit, as a victim of the mother's whims, expresses a desire that falls outside the scope of the phallic principle and is, in itself, perverse. This explains why such a child has no other choice but to become divided and nonsensical.

Key words: Maternal perversion, behavioral disorders in a child, aphallic relationship, psychopathy

(Perversión materna y transtornos del comportamiento del niño: el ejemplo de Dylan, objeto del capricho de su madre)

A partir de una situación clínica, analizamos como los trastornos conductuales del niño responden a una postura psíquica perversa de su madre. Destacamos el concepto de fantasma materno edipiano transformado en acto y exploramos la posición del hijo como fetiche. Demonstramos que el estatus de los actos del sujeto atrapado en la sinrazón y el engaño, transformado en objeto del capricho materno, designa un deseo inscrito fuera de la ley fálica y que tal inscripción es, de por sí, perversa. Eso explica que, entonces, el hijo no tiene otra salida que la de convertirse en alguien clivado, sin sentido.

Palabras clave: Perversión materna, transtornos del comportamiento del niño, relación a-fálica, psicopatía

(Mütterliche Perversion und Verhaltensstörungen des Kindes: ams Beispiel von Dylan, Objekt der Launen seiner Mutter)

Ausgehend von einem klinischen Fall untersuchen wir, inwiefern die angeblichen Verhaltensprobleme des Kindes eigentlich eine Reaktion auf eine perverse psychische Haltung der Mutter sind. Es wird die Auffassung des mütterlichen Ödipusphantoms hervorgehoben, das in Handlung umgesetzt wird. Es wird hinterfragt, inwiefern die Rolle des Kindes als Fetischgegenstand zu sehen ist. Es wird aufgezeigt, dass insbesondere das Statut der Handlungen des Subjektes, aus der Perspektive des Sinnesfremden und der Täuschung betrachtet, Objekt der mütterlichen Launen, auf einen Wunsch deuten, der außerhalb des phallischen Gesetzes liegt, und dass diese Inschrift pervers in sich ist. Sie 


\section{ARTIGOS}

erklärt, dass das Kind in diesem Moment keine andere Wahl hat, als sich selbst zu splitten und sinnesfremd zu werden.

Schlüsselwörter: Mütterliche Perversion, Verhaltensstörungen des Kindes, MutterVersion, aphallische Beziehung, Psychopathie

Citação/Citation: Martin-Lavaud, V. (2014, setembro). Perversion maternelle et troubles du comportement de l'enfant: l'exemple de Dylan, objet du caprice de sa mère. Revista Latinoamericana de Psicopatologia Fundamental, 17(3-Suppl.), 706-719.

Editor do artigo/Editor: Prof. Dr. Manoel Tosta Berlinck

Recebido/Received: 15.3.2014/ 3.15.2014 Aceito/Accepted: 15.4.2014 / 4.15.2014

Copyright: (C) 2009 Associação Universitária de Pesquisa em Psicopatologia Fundamental/ University Association for Research in Fundamental Psychopathology. Este é um artigo de livre acesso, que permite uso irrestrito, distribuição e reprodução em qualquer meio, desde que o autor e a fonte sejam citados / This is an open-access article, which permits unrestricted use, distribution, and reproduction in any medium, provided the original author and source are credited.

Financiamento/Funding: A autora declara não ter sido financiada ou apoiada / The author have no support or funding to report.

Conflito de interesses/Conflict of interest: A autora declara que não há conflito de interesses / The author has no conflict of interest to declare.

\section{Virgine Martin-LaVaud}

Psychologue; Psychanalyste; Dr. en psychopathologie; Membre de l'equipe de recherche EA 4050, Université catholique de l'Ouest, Angers, France.

66 rue du Dr Guichard

49000 Angers

France

e-mail: martinlavaud@wanadoo.fr 